\section{ABC Transporter Genes and Methotrexate Response in Rheumatoid Arthritis}

\section{To the Editor:}

In a recent issue, de Rotte, et al ${ }^{1}$ reported that 3 single-nucleotide polymorphisms (SNP) in 3 key methotrexate (MTX) transporter genes were determinants of response to MTX in patients with juvenile idiopathic arthritis (JIA). These investigators are to be commended for their efforts in a field, juvenile arthritis, where there is a relative scarcity of data on MTX pharmacogenetics, compared to adult rheumatoid arthritis (RA). Their study examined the association between $21 \mathrm{SNP}$ in 13 genes involved in MTX cellular transport and polyglutamation, and found 3 SNP in $S L C 19 A 1, A B C B 1$, and $A B C C 3$, all 3 transporter genes, were associated with MTX efficacy in a cohort of 287 patients with JIA.

Our study in adult patients with $\mathrm{RA}^{2}$ using a retrospective cross-validation approach demonstrated that $4 \mathrm{SNP}$, one in $A B C B 1$ and 3 in $A B C C 2$, were associated with an increased risk of MTX toxicity in our training cohort. These SNP were predictors of individual toxicities such as alopecia and gastrointestinal and hepatic toxicity, except the $A B C B 1$ SNP, which was a marker of overall toxicity. These associations differed by race, with unique genetic markers in whites and African Americans with RA. In our validation cohort, one of the $A B C C 2$ SNP (an intronic SNP) influenced MTX toxicity-related time to discontinuation or dose decrease; again, this was race-specific, and this effect was seen only in white patients with $\mathrm{RA}^{2}$.

It would be interesting to know whether similar associations were found in the cohort studied by de Rotte, et al. Because that was a prospective, longitudinal cohort followed for 1 year, data on any associations (or lack thereof) between the transporter gene SNP and MTX toxicity would be valuable, if ascertainable. Because it appears that laboratory measurements and reasons for discontinuation of MTX in the cohort were documented, the question of whether there were any associations between these SNP and MTX toxicity and toxicity-related discontinuation could be answered. It is important to note that this was a pediatric cohort, and such data, if available, will give important insights into whether MTX pharmacogenetic associations are comparable in pediatric and adult RA populations, a subject on which there is a paucity of data to date ${ }^{3}$. Unfortunately, the effect of race on such associations cannot be determined using this cohort, as it was racially homogeneous.

ABC transporter gene SNP appear to determine MTX response both in adult RA and in JIA, but affect different outcomes, i.e., toxicity in adult RA and efficacy in JIA. This is an area of MTX pharmacogenetics that needs further investigation.

PRABHA RANGANATHAN, MD, MS, Division of Rheumatology, Washington University School of Medicine, 660 S. Euclid Avenue, Campus Box 8045, St. Louis, Missouri 63110, USA.

E-mail:prangana@dom.wustl.edu

\section{REFERENCES}

1. de Rotte MC, Bulatovic M, Heijstek MW, Jansen G, Heil SG, van Schaik RH, et al. ABCB1 and ABCC3 gene polymorphisms are associated with first-year response to methotrexate in juvenile idiopathic arthritis. J Rheumatol 2012;39:2032-40.

2. Ranganathan P, Culverhouse R, Marsh S, Mody A, Scott-Horton TJ, Brasington R, et al. Methotrexate (MTX) pathway gene polymorphisms and their effects on MTX toxicity in Caucasian and African American patients with rheumatoid arthritis. J Rheumatol 2008;35:572-9.

3. Goldman J, Becker ML, Jones B, Clements M, Leeder JS. Development of biomarkers to optimize pediatric patient management: What makes children different? Biomark Med 2011;5:781-94.

J Rheumatol 2013;40:4; doi:10.3899/jrheum.121361 